01

\title{
Диссоциативная рекомбинация в послесвечении барьерного разряда В неоне низкого давления. Заселение атомов конфигурации $2 p^{5} 3 d$
}

\author{
(C) B.A. Иванов \\ Санкт-Петербургский государственный университет, \\ 198504 Санкт-Петербург, Россия \\ e-mail: v.a.ivanov@spbu.ru
}

Поступила в редакцию 15.04.2021 г.

В окончательной редакции 14.05.2021 г.

Принята к публикации 06.09.2021 г.

На основе спектроскопического исследования распадающейся неоновой плазмы, создаваемой диэлектрическим барьерным разрядом в цилиндрической трубке при давлении 0.1-40 Torr, выполнен анализ диссоциативной рекомбинации молекулярных ионов $\mathrm{Ne}_{2}^{+}$с электронами как механизма образования возбужденных атомов. Показано, что при плотности электронов менее $5 \cdot 10^{10} \mathrm{~cm}^{-3}$ этот процесс является доминирующим источником заселения уровней конфигурации $2 p^{5} 3 d$ при давлениях $P_{\mathrm{Ne}} \geq 0.6$ Torr. C уменьшением давления неона оптические свойства распадающейся плазмы в большей степени формируются за счет ударно-радиационной рекомбинации ионов $\mathrm{Ne}^{+}$. Обнаружено значительное изменение относительных интенсивностей линий $3 d \rightarrow 3 p$ переходов в послесвечении с изменением давления неона, отражающее влияние неупругих столкновений на формирование спектра излучения плазмы в ближней инфракрасной области. По результатам измерений, проведенных при давлении 0.65 Torr, найдено распределение потока диссоциативной рекомбинации по $3 d_{j}$ уровням атома неона, не искаженное столкновительным ,перемешиванием“ возбужденных состояний. Сопоставление этих данных с измерениями в ближней ультрафиолетовой области, содержащей лини $4 p \rightarrow 3 s$ переходов, указывает на необходимость учета каскадных $4 p \rightarrow 3 d$ переходов для корректного решения задачи о конечных продуктах диссоциативной рекомбинации.

Ключевые слова: диэлектрический барьерный разряд, молекулярные ионы, диссоциативная рекомбинация, распадающаяся плазма, элементарные процессы, константа скорости.

DOI: $10.21883 / \mathrm{OS} .2021 .11 .51633 .2177-21$

\section{Введение}

Неизменный интерес исследователей к процессу диссоциативной рекомбинации (DR) молекулярных ионов, впервые предложенному авторами [1] для объяснения явлений с участием заряженных частиц в ионосфере, объясняется двумя обстоятельствами. С одной стороны, этот механизм деионизации определяет свойства широкого круга лабораторных плазменных объектов и является необходимым звеном в моделях звездной и планетной атмосфер [2]. С другой стороны, несмотря на долгую историю изучения процесса, обнаруживаются ранее неизвестные проявления DR даже в случае простейших молекулярных ионов, таких как $\mathrm{H}_{2}^{+}$[3] или $\mathrm{HeNe}^{+}$[4], что стимулирует развитие новых подходов к расчету его основных характеристик - сечений и констант скорости [5]. Наиболее подробно обсуждаемый процесс изучен в плазме инертных газов, а наибольшее количество работ посвящено рекомбинации ионов $\mathrm{Ne}_{2}^{+}$. Реакция DR в случае неона описывается уравнением

$$
\mathrm{Ne}_{2}^{+}(v)+e \rightarrow\left(\mathrm{Ne}_{2}^{*}\right)_{\text {unstable }} \stackrel{\alpha_{j}}{\longrightarrow} \mathrm{Ne}^{*}(j)+\mathrm{Ne}+E_{j}^{\mathrm{kin}},
$$

где $\alpha_{j}$ - парциальные коэффициенты рекомбинации, пропорциональные вероятности образования возбужденных атомов в конечном состоянии $j$. Процесс (1) является многоканальным по своей природе как во входных, так и в выходных каналах. В рекомбинации могут участвовать ионы в различных колебательновращательных состояниях $v$, и в результате реакции появляются атомы в ограниченном наборе возбужденных состоянии $j$, уносящие вместе с атомом в основном состоянии энергию диссоциации. Очевидно, что парциальные коэффициенты, как и энергия диссоциации, должны характеризоваться как минимум двумя индексами $v$ и $j$. Однако мы вынуждены упростить анализ, рассматривая проблему в рамках предположения, что распределение ионов по внутренним состояниям не зависит от условий эксперимента. Причинами являются, во-первых, отсутствие надежных экспериментальных данных по кинетике возбужденных молекулярных ионов в плазме и, вовторых, доминирующее в литературе после расчетов [6] мнение о чрезвычайно высокой скорости колебательной релаксации молекулярных ионов при столкновениях с атомами, согласно которым константа скорости $k_{v}$ процесса превышает $10^{-10} \mathrm{~cm}^{3} / \mathrm{s}$. При давлении газа $\approx 1$ Torr время релаксации $\tau_{r}<1 \mu \mathrm{s}$, что как минимум на два порядка меньше времени рекомбинации $1 / \alpha_{s}[e]$ в условиях нашего эксперимента (концентрация электронов $[e]<5 \cdot 10^{10} \mathrm{~cm}^{-3}$ ), если использовать константу скорости DR [7]

$$
\alpha_{s}=1.7 \cdot 10^{-7}\left(300 / T_{e}\right)^{0.43}\left(\mathrm{~cm}^{3} / \mathrm{s}\right) \text {. }
$$


Учитывая вышеизложенное, $\alpha_{s}$ можно представить суммой парциальных коэффициентов: $\alpha_{s}=\sum \alpha_{j}$. В этом приближении коэффициенты $\alpha_{s}$ и $\alpha_{j}$ полностью характеризуют процесс рекомбинации и позволяют выразить потоки $F_{j}$ заселения возбужденных уровней атомов следующим образом:

$$
F_{j}=\alpha_{j}\left[\mathrm{Ne}_{2}^{+}\right][e] .
$$

Спектроскопические наблюдения [8-14] послесвечения тлеющего разряда постоянного (DC) или высокочастотного (HF) тока в инертных газах (исключением является гелий [15]) показали, что DR заселяет возбужденные уровни $n p^{5}(n+1) p, n p^{5}(n+1) d$ и $n p^{5}(n+2) p(n-$ главное квантовое число невозбужденного электрона). Излучение на переходах с более высоких уровней, в работах, где оно было замечено [8-10,14], авторы связывали с ударно-радиационной рекомбинацией (CRR) ионов $\mathrm{Ne}^{+}$:

$$
\mathrm{Ne}^{+}+e+e \stackrel{\alpha_{\mathrm{cr}}}{\longrightarrow} \mathrm{Ne}^{+}+e
$$

( $\alpha_{\mathrm{cr}}$ - коэффициент CRR).

B соответствии с механизмом процесса [16,17], первичными продуктами захвата электрона ионом при тройном столкновении (3) являются высоковозбужденные состояния атома, а распределение населенностей по атомным уровням формируется в результате конкуренции столкновительных и радиационных переходов

$$
\begin{gathered}
\mathrm{Ne}_{k}^{+}+e \Leftrightarrow \mathrm{Ne}_{j}^{+}+e, \\
\mathrm{Ne}_{k}^{+} \rightarrow \mathrm{Ne}_{j}^{+}+h v,
\end{gathered}
$$

так что в плазме с атомарными ионами возбужденные атомы могут появляться в любых состояниях, а излучение, вызванное CRR, переносится всеми линиями послесвечения.

В экспериментах по измерению коэффициентов $\alpha_{j}$ этот ,фон“ устранялся выбором остаточно высокого давления газа (например, $P_{\mathrm{Ne}}=20$ Torr [11], 10 Torr [13]), когда ионный состав плазмы таков, что $\left[\mathrm{Ne}_{2}^{+}\right] \gg\left[\mathrm{Ne}^{+}\right]$ из-за быстрой конверсии атомарных ионов в молекулярные

$$
\mathrm{Ne}^{+}+\mathrm{Ne}+\mathrm{Ne} \stackrel{\beta}{\rightarrow} \mathrm{Ne}_{2}^{+}+\mathrm{Ne}
$$

$\left(\beta \approx 5 \cdot 10^{-32} \mathrm{~cm}^{6} / \mathrm{s}[18]\right.$ - константа скорости конверсии).

Однако данные цитированных работ едва ли отражают реальное распределение потока (2) по уровням $j$ изза неопределенности, которую могут внести неупругие атомно-атомные столкновения

$$
\mathrm{Ne}_{k}^{*}+\mathrm{Ne} \Leftrightarrow \mathrm{Ne}_{j}^{*}+\mathrm{Ne} .
$$

Об этом свидетельствуют результаты экспериментов, проведенных в широком диапазоне изменения давления $[12,14,19]$, которые однозначно указывают на участие столкновений (7) в формировании спектра послесвечения при давлениях выше 1 Torr. Очевидно, что корректные данные о значениях $\alpha_{j}$ можно получить только при $P_{\mathrm{Ne}}<1$ Torr. Исследование DR молекулярных ионов в спектроскопическом эксперименте при столь низких давлениях пока ограничивается данными [14,20]. В работе [14] относительные населенности $4 p$-уровней атома неона измерены в диапазоне давлений 150-0.2 Torr, и было показано, что вплоть до $P_{\mathrm{Ne}} \approx 0.6$ Torr при плотности электронов $[e] \leq 5 \cdot 10^{10} \mathrm{~cm}^{-3}$ можно, основываясь на различии временных характеристик интенсивностей спектральных линий $J(t)$ и их реакции на импульсный „нагрев“ электронов в послесвечении, надежно разделить потоки DR и CRR и найти относительные значения коэффициентов $\alpha_{j}$. В этих условиях конверсия атомарных ионов (6) подавлена, а другие механизмы образования молекулярных ионов неэффективны [20], так что их плотность мала $\left(\left[\mathrm{Ne}_{2}^{+}\right] \ll\left[\mathrm{Ne}^{+}\right]\right)$, и излучение распадающейся плазмы оказывается близким к порогу возможностей регистрации методом многоканального счета фотонов. Однако известное свойство DR - высокая селективность процесса по сравнению c C-RR и как минимум на два порядка более высокий коэффициент рекомбинации (при $[e] \approx 5 \cdot 10^{10} \mathrm{~cm}^{-3}$ ) $[16,17]$ делают задачу разрешимой. Селективность DR особенно ярко проявляется при заселении уровней конфигурации $2 p^{5} 4 p$. Согласно результатам [14], доступными для DR при близких к комнатной температурах электронов оказываются только те уровни атома неона, которые расположены в шкале энергии ниже $4 p_{4}{ }^{1}$ ( $3 p_{3}$ в обозначениях Пашена), т.е. $4 p_{5}-4 p_{10}$. Для полноты картины послесвечения неона отметим, что, насколько нам известно, в настоящее время отсутствуют данные о роли DR в заселении уровней конфигурации $2 p^{5} 4 s$.

Целью данной работы было решение задачи о распределении потока DR по $3 d$-уровням атома неона при минимально возможном давлении неона.

\section{Постановка эксперимента}

В качестве источника плазмы использовался диэлектрический барьерный разряд (DBD) в стеклянной цилиндрической трубке длиной $20 \mathrm{~cm}$ и внутренним диаметром $3.9 \mathrm{~cm}$. Преимущества исследования DR в послесвечении такого разряда и детали эксперимента описаны в [20]. Частота разряда составляла $80-200 \mathrm{~Hz}$, наблюдения проводились вдоль оси разрядной трубки. Давление неона 0.1-40 Torr, плотность электронов в послесвечении на оси трубки $[e] \leq 5 \cdot 10^{10} \mathrm{~cm}^{-3}$. Величина $[e]$ оценивалась по скорости разрушения атомов в резонансном состоянии $2 p^{5} 3 s\left({ }^{3} P_{1}\right)$ электронами в послесвечении [20]. Температура стенки трубки во всех условиях была равна комнатной. Световые потоки регистрировались многоканальным счетчиком фотонов, спектральная чувствительность $S(\lambda)$ установки

\footnotetext{
1 Здесь и далее мы используем простейший способ обозначения уровней в соответствии с их положением в шкале энергий: $4 p_{1}$ верхний уровень конфигурации $2 p^{5} 4 p, 4 p_{10}$ - нижний. Аналогично $3 p_{1}-3 p_{10}, 3 d_{1}-3 d_{12}$.
} 


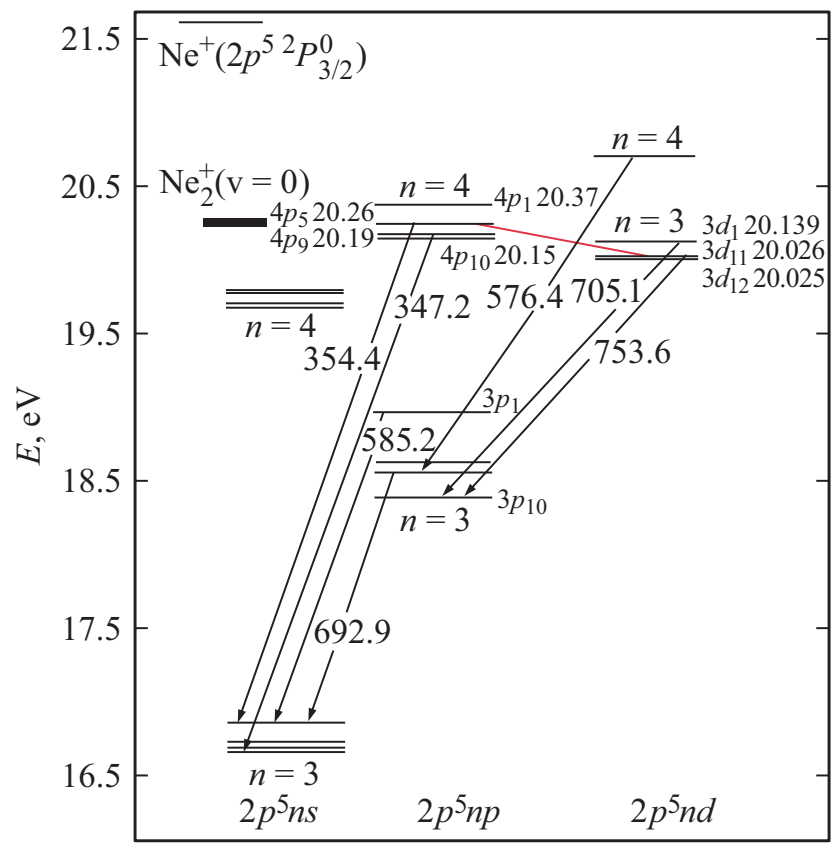

Рис. 1. Схема возбужденных уровней и линий атома неона (в $\mathrm{nm})$ и энергии атомарных и молекулярных ионов. Положение колебательного уровня $v=0$ указано в соответствии с результатами [3].

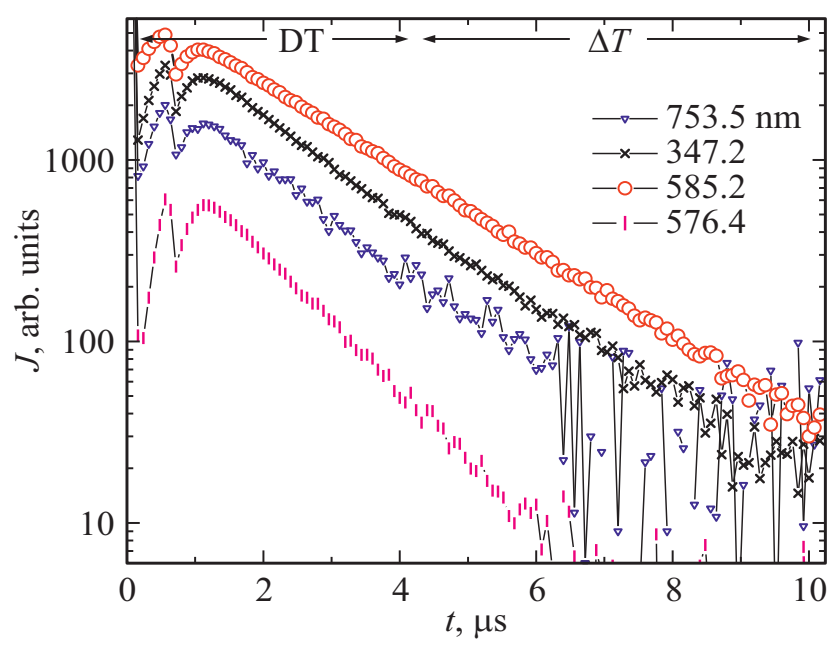

Pис. 2. Послесвечение DBD на оси трубки. $P_{\mathrm{Ne}}=0.65$ Torr. $[e](t=1.5 \mathrm{~ms}) \approx 4 \cdot 10^{10} \mathrm{~cm}^{-3}$. Для удобства представления результатов числа фотоэлектронов умножены на следующие коэффициенты: $585.2 \mathrm{~nm}-1,576.4 \mathrm{~nm}-1,347.2 \mathrm{~nm}-3.5$, $753.5 \mathrm{~nm}-10$.

определялась в диапазоне 345-850 nm по излучению источника непрерывного спектра LS-1-CAL. Как и в работе [14], для идентификации источника заселения возбужденных уровней мы анализировали реакцию излучения распадающейся плазмы на импульсный подогрев электронов. Отличие от [14] лишь в том, что в качестве источника нагрева в данной работе мы воспользовались высокочастотным электрическим полем, как это описано в [20]. Некоторые из исследованных линий и соответствующие им возбужденные уровни показаны на рис. 1. Мы использовали два метода измерения интенсивностей линий в послесвечении. При давлениях выше нескольких Torr, т. е. при относительно ярких линиях атома неона, регистрировались спектры послесвечения в области 700-850 $\mathrm{nm}$. В этом режиме схема счета фотонов суммировала импульсы фотоэлектронного умножителя в течение строб-импульса $\Delta T$, параметры которого - длительность и задержка DT выбирались на основании наблюдений $J_{j k}(t)$ с требуемым временным разрешением. На рис. 2,3 показан пример таких измерений. Данные рис. 2 подтверждают идентичность изменения во времени потоков фотонов на переходах $3 p \rightarrow 3 s, 3 d \rightarrow 3 p$ и $4 p \rightarrow 3 s$ в стадии достаточно далекого послесвечения и их четкое отличие от интенсивности $J_{576.4}(t)$ линии $576.4 \mathrm{~nm}$, верхний $4 d-$ уровень которой лежит намного выше основного $v=0$ колебательного уровня иона $\mathrm{Ne}_{2}^{+}$(рис. 1) и по этой причине заселяется исключительно за счет CRR ионов $\mathrm{Ne}^{+}$(3). Это отличие подтверждается также и реакцией интенсивностей на импульсный подогрев электронов. В качестве опорной линии $3 p \rightarrow 3 s$ переходов была выбрана линия 585.2 нм, излучение которой переносит значительную долю потока DR [12]. Также видно, что помещая строб в достаточно позднее послесвечение, можно минимизировать вклад CRR (рис. 2), даже если он заметен на начальной стадии. Фрагмент спектра послесвечения, показанный на рис. 3, поясняет методику вычисления интенсивностей линий. С помощью программы Origin мы, аппроксимируя $J(\lambda)$ функцией Гаусса (сплошная кривая на рис. 3), находили амплитуду в максимуме и принимали ее равной интенсивности линии. Причина относительно низкого спектрального разрешения - необходимость работы с широкими входными и выходными щелями монохроматора для увеличения

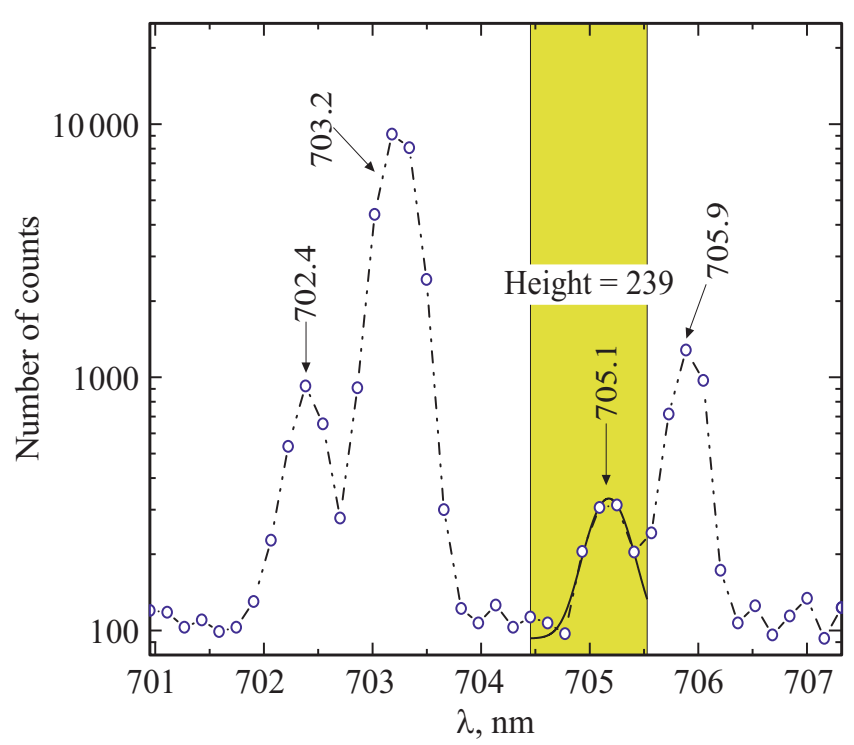

Pис. 3. Фрагмент спектра послесвечения при $P_{\mathrm{Ne}}=10$ Torr. 
Таблица 1. Параметры спектральных линий $3 d \rightarrow 3 p$ переходов. В скобках - данные для резонансных линий, в нижней строке - для линии 345.4 нм перехода $4 p_{5}-3 d_{11}$

\begin{tabular}{c|c|c|c|c|c}
\hline $\begin{array}{c}\text { Upper } \\
\text { level }\end{array}$ & $\begin{array}{c}\text { Energy, } \\
\mathrm{eV}\end{array}$ & $\lambda_{j k}, \mathrm{~nm}$ & $A_{j k}, 10^{6} \mathrm{~s}^{-1}$ & $A_{j}=\Sigma_{v}\left(A_{j k}\right)$ & $S\left(\lambda_{j k}\right)$ \\
\hline $3 d_{1}$ & 20.1394 & $705.1(61.56)$ & $2.38(40.3)$ & $44.4(84.7)$ & 0.89 \\
$3 d_{2}$ & 20.1375 & 705.9 & 7.46 & 48.2 & 0.89 \\
$3 d_{3}$ & 20.1363 & 794.3 & 3.82 & 47.7 & 0.25 \\
$3 d_{4}$ & 20.1361 & 813.6 & 11.8 & 48.6 & 0.17 \\
$3 d_{5}$ & 20.0484 & 830.0 & 14 & 46.6 & 0.11 \\
$3 d_{6}$ & 20.0482 & 841.8 & 15.6 & 46.5 & 0.088 \\
$3 d_{7}$ & 20.0404 & $747.2(61.87)$ & $2.96(80)$ & $41.8(121.8)$ & 0.58 \\
$3 d_{8}$ & 20.0367 & 748.9 & 23.1 & 50.5 & 0.57 \\
$3 d_{9}$ & 20.0349 & 849.5 & 39 & 48.2 & 0.068 \\
$3 d_{10}$ & 20.0346 & 837.7 & 50 & 50 & 0.096 \\
$3 d_{11}$ & 20.0264 & $753.6(61.91)$ & $30.6(26.6)$ & $50.5(77.1)$ & 0.61 \\
$3 d_{12}$ & 20.0246 & 754.4 & 38.7 & 53.8 & 0.61 \\
$4 p_{5}$ & 20.2592 & 345.4 & 36.8 & 112 & 0.28
\end{tabular}

светового потока. При более низких давлениях более продуктивным оказалось длительное (до нескольких часов) измерение двух сигналов: первого - интенсивности $J_{j k}(t)$ и второго - сигнала $J_{D}(t)$ с настройкой монохроматора на ближайший спектральный интервал, свободный от линий. Например, в случае линии $705.1 \mathrm{~nm}$ монохроматор перестраивался на $704.5 \mathrm{~nm}$. (рис. 3). Вычитание $J_{j k}(t)-J_{D}(t)$ позволяло учесть не только „темновые“ фотоэлектроны, но и слабую засветку от рассеянного в монохроматоре света сильных линий переходов $3 p-3 s$. Для сравнения световых потоков вычислялись суммы чисел фотоэлектронов в каналах счетчика фотонов, соответствующих интервалу $\Delta T$ (рис. 2).

\section{Обсуждение результатов}

Список линий, по которым определялись парциальные коэффициенты рекомбинации и их характеристики представлены в табл. 1. Из этих данных мы видим, что конфигурация $2 p^{5} 3 d$ состоит из двух групп близкорасположенных уровней, отвечающих различным моментам атомного остатка, расстояние между которыми меньше тепловой энергии $k T_{a} \approx 0.03 \mathrm{eV}$. Это еще раз подчеркивает необходимость выбора в таком эксперименте крайне низких давлений. Минимальное давление, при котором нам удалось разделить потоки DR и CRR, составило $P_{\mathrm{Ne}} \approx 0.6$ Torr. При вычислении парциальных коэффициентов рекомбинации использовалось следующее достаточно очевидное соотношение:

$$
\alpha_{j}=J_{j k} A_{j} /\left(A_{j k} S\left(\lambda_{j k}\right)\right),
$$

где $J_{j k}$ - найденные одним из описанных выше способов интенсивности спектральных линий. В вычислениях мы использовали вероятности переходов $A_{j k}$ из NIST database, представленные в табл. 1, где $A_{j}-$ сумма вероятностей по всем разрешенным переходам с уровня $j$.
Для трех уровней $-3 d_{1}, 3 d_{7}$ и $3 d_{11}$ мы приводим два значения $A_{j}$ с учетом (в скобках) и без учета переходов в основное состояние атома неона, разрешенных для этих уровней. Наличие резонансных переходов вносит неопределенность в вычисления $\alpha_{j}(8)$, устранение которой требует анализа эффекта „пленения“ излучения в условиях эксперимента. Решение этой задачи $[21,22]$ для различных геометрий и механизмов уширения спектральных линий показывает, что для наших условий (плотность атомов более $10^{16} \mathrm{~cm}^{-3}$ ) неон оказывается оптически плотным $\left(\kappa_{0} R \gg 1, \kappa_{0}-\right.$ коэффициент поглощения в центре линии, $R$ - радиус разрядной трубки), так что резонансное излучение при вычислении вероятностей $A_{j}$ можно не учитывать. Однако в нашей ситуации проблема усложняется из-за специфики профилей спектральных линий в послесвечении, связанном с диссоциативной рекомбинацией. В соответствии с (1) в результате реакции возникают возбужденные атомы с кинетической энергией $\delta E_{j}^{\mathrm{kin}} / 2$, которая значительно превышает тепловую энергию $k T_{a}$ атомов при $T_{A}=300 \mathrm{~K}$. Сравнивая энергии основного колебательного уровня иона $\mathrm{Ne}_{2}^{+}$и уровней $3 d_{j}$ (рис. 1), находим, что минимальная кинетическая энергия (соответствует заселению уровня $\left.3 d_{1}\right) \Delta E_{j}^{\mathrm{kin}} / 2 \approx 0.06 \mathrm{eV}$, максимальная (для $3 d_{11}$ ) $\Delta E_{j}^{\mathrm{kin}} / 2 \approx 0.12 \mathrm{eV}$. Спектральная ширина линий таких атомов из-за эффекта Доплера оказывается намного больше ширины линии поглощения атомов в основном состоянии $[10,24]$. В этих исследованиях авторы наблюдали в послесвечении неона при давлениях менее 3 Torr линии $3 p \rightarrow 3 s$ переходов в 3 раза более широкие, чем в разряде. Для линий $3 d \rightarrow 3 p$ такие наблюдения не проводились, но из сравнения значения $\Delta E_{j}^{\mathrm{kin}} / 2 \approx 0.12 \mathrm{eV}$ и энергии $\Delta E_{3 p}^{\mathrm{kin}} / 2 \approx 0.63 \mathrm{eV}$ для уровня $3 p_{1}$ (рис. 1) ясно, что уширение будет гораздо менее значительным. С точки зрения задач данной работы наибольший интерес представляют измерения, выполненные при давлениях менее 1 Torr, когда форму линии поглощения можно считать близкой к доплеровской. В этом приближении нарушение условия $\kappa_{0} R \gg 1$, по нашим оценкам, может иметь место только на крыльях резонансной линии $61.91 \mathrm{~nm}$ (верхний уровень $3 d_{11}$ ), переносящих незначительную долю интегрального излучения линии. Следуя этим соображениям, мы не учитывали резонансные переходы при вычислении коэффициентов ветвления. Заметим, что учесть их нетрудно, пересчитав коэффициенты ветвления при наличии точного решения задачи.

Основные результаты работы представлены на рис. 4 в виде потоков квантов $F(j)=F_{j} / F_{12}$, излучаемых $3 d$ уровнями, отнесенных к потоку $F_{12}$ с нижнего уровня при различных давлениях неона. Видно существенное изменение относительных величин потоков с уменьшением давления, отчетливо указывающее на увеличение вклада верхних уровней в спектр послесвечения. Неопределенность величин $F(j)$, обусловленную малостью световых потоков при давлениях 1.25 и 0.65 Torr, 


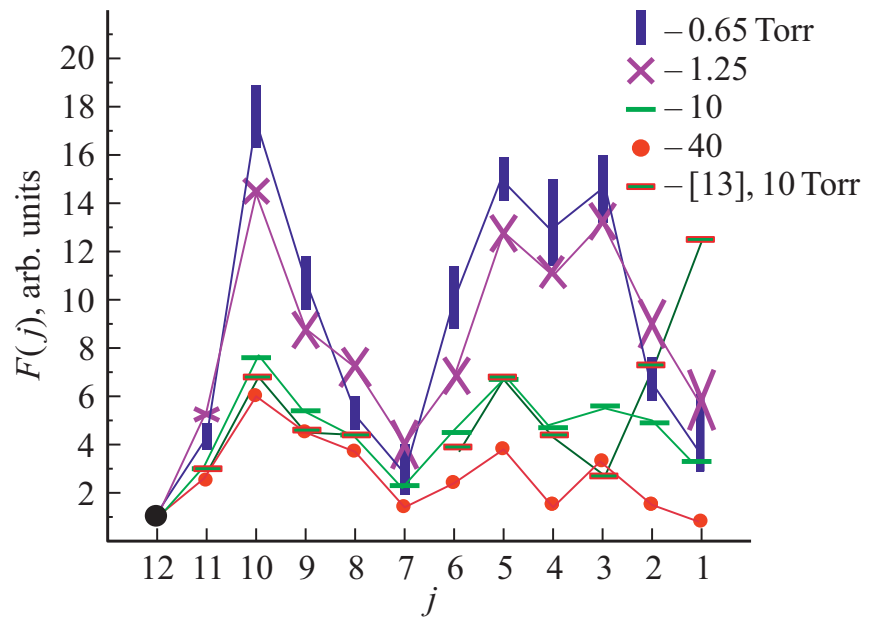

Рис. 4. Потоки квантов с $3 d_{j}$-уровней атома неона в послесвечении, отн. ед.

мы попытались приближенно отобразить вертикальным размером соответствующих символов. Важно отметить, что переход от 1.25 к 0.65 Torr приводит лишь к небольшой деформации распределения потока квантов, что, очевидно, указывает на стабилизацию $F(j)$ как функции давления и в русле логики работы может быть связано с уменьшением влияния неупругих столкновений (7) при малом давлении. Это означает, что величины $F(j)$, относящиеся к $P_{\mathrm{Ne}}=0.65$ Torr, представляют распределение потока DR по уровням конфигурации $2 p^{5} 3 d$, т.е. они пропорциональны относительным величинам парциальных коэффициентов $\alpha_{j}$. Данные для $3 d_{1}, 3 d_{7}$ и $3 d_{11}$ получены в предположении, что резонансное излучение пленено, т. е в вероятностях $A_{j}$ учтены только переходы $3 d \rightarrow 3 p$.

Данные нашей работы мы сравнили с единственными имеющимися в литературе результатами подобного эксперимента [13], в котором найдены относительные населенности $N_{j}$ уровней $3 d_{J}$ атома неона в послесвечении высокочастотного разряда в неоне при давлении 10 Torr. В расчетах авторы [13] использовали вероятности переходов, предложенные в [24]. По величинам $N_{j}$ и вероятностям переходов [24] мы восстановили относительные потоки $F(j)$. В [13] отсутствуют данные для уровней $3 d_{12}$ и $3 d_{7}$, поэтому для наглядности сравнения на рис. 4 приводим вычисленные потоки, совместив их с нашими данными для $3 d_{11}$ при давлении 10 Torr. Видно, что, в основном, результаты [13] близки к нашим, за исключением верхних уровней $3 d_{2}$ и $3 d_{1}$. Нам трудно судить о степени корректности этих данных. Отметим только, что они получены по интенсивностям линий 705.9 и $867.9 \mathrm{~nm}$, тогда как мы обратились к линиям 705.9 и $705.1 \mathrm{~nm}$, для которых квантовый выход фотоэлектронного умножителя одинаков и поэтому исключены ошибки, связанные с определением чувствительности схемы регистрации излучения в широком диапазоне длин волн. Из фрагмента спектра рис. 3 и
Таблица 2. Парциальные коэффициенты рекомбинации (в \%) на уровни $3 d$ и $4 p$

\begin{tabular}{c|c|c|c|c|c|c|c|c|c|c|c|c}
\hline$j$ & 12 & 11 & 10 & 9 & 8 & 7 & 6 & 5 & 4 & 3 & 2 & 1 \\
\hline$\alpha_{3 d j}$ & 0.01 & 0.04 & 0.17 & 0.1 & 0.05 & 0.03 & 0.1 & 0.14 & 0.13 & 0.14 & 0.06 & 0.04 \\
$\alpha_{4 p j}$ & & & 0.1 & 0.22 & 0.18 & 0.17 & 0.22 & 0.11 & & & &
\end{tabular}

вероятностей переходов табл. 1, мало отличающихся от использованных авторами [13], следует обратное предложенному в [13] отношение потоков $F(j=2) / F(j=1)$.

В табл. 2 мы разместили парциальные коэффициенты рекомбинации $\alpha_{j}^{\text {rel }}=\alpha_{j} / \sum \alpha_{j}$, найденные по представленным на рис. 4 данным. Эти величины отражают вклад потока рекомбинации на уровень $3 d_{j}$ в полный поток рекомбинации на уровни $2 p^{5} 3 d$. Вместе с аналогичными данными по результатам [14] для уровней $4 p_{5}-4 p_{10}$ конфигурации $2 p^{5} 4 p$ (также представлены в табл. 2) они дают полное представление о DR молекулярных ионов $\mathrm{Ne}_{2}^{+}$как источнике заселения возбужденных уровней указанных конфигураций.

Определение парциальных коэффициентах DR должно сопровождаться анализом роли каскадных переходов. Например, по данным [13], заселение $3 p$-уровней атома неона почти на четверть обусловлено каскадами $3 d \rightarrow 3 p$. Подобная задача не стоит только в отношении $4 p$-уровней, шесть нижних из которых [14] связаны в послесвечении непосредственно с DR (1). Для выяснения вклада каскадов в заселение $3 d$-уровней необходимы данные о линиях $4 p \rightarrow 3 d$ переходов, расположенных в далекой инфракрасной области спектра. В NIST Database мы нашли единственную линию $5327 \mathrm{~nm}$ (на рис. 1 показан соответствующий переход $\left.4 p_{5} \rightarrow 3 d_{11}\right)$ с вероятностью перехода $\left.A_{p d}=1.74 \cdot 10^{5} \mathrm{~s}^{-1}\right)$. Пользуясь полным набором данных по интенсивностям линий и вероятностям $4 p \rightarrow 3 s$ переходов в аналогичных условиях [14], и данными рис. 2, нетрудно оценить вклад потока $4 p_{5} \rightarrow 3 d_{11}$ в излучение линии $753.6 \mathrm{~nm}$. При $t \geq 5 \mathrm{~ms}$ от начала послесвечения, когда исключено влияние ударнорадиационной рекомбинации, он составляет $\approx 10 \%$. Подчеркнем, что это вклад только одного, причем наименее населенного в послесвечении [14] уровня конфигурации $2 p^{5} 4 p$. Если принять во внимание, что в силу правил отбора разрешены переходы на $3 d_{11}$ также и с уровней $4 p_{6}$, $4 p_{7}, 4 p_{8}$ и $4 p_{10}$, суммарная населенность которых на порядок превышает населенность $4 p_{5}$, то правомерен вопрос о возможности прямого заселения уровней $3 d$ при DR ионов $\mathrm{Ne}_{2}^{+}$. Для ответа на него необходим полный набор данных о вероятностях более чем пятидесяти разрешенных $4 p \rightarrow 3 d$ переходов, которыми мы не располагаем. Отметим, что если именно они являются источником заселения уровней $3 d$, вопрос о контурах спектральных линий в задаче о пленении излучения перестает быть значимым.

Вследствие неопределенности с каскадными переходами коэффициенты $\alpha_{3 d j}$ (табл. 2), в отличие от $\alpha_{4 p j}$, 
могут быть названы парциальными коэффициентами рекомбинации лишь с известной оговоркой. Заметим, что задача по определению парциальных коэффициентов и выяснению роли каскадных переходов возникает не только в отношении инертных газов, она актуальна для любого типа плазмы, содержащей молекулярные ионы. Особый интерес эти данные представляют для прогнозирования свойств активных сред на плазме с молекулярными ионами [25]. Они также могут использоваться в качестве реперных данных для тестирования теоретических моделей (например, [26,27]), претендующих на адекватное распределения потока рекомбинации по выходным каналам процесса.

\section{Выводы}

Методами оптической эмиссионной спектроскопии найдено распределение потока диссоциативной рекомбинации молекулярных ионов $\mathrm{Ne}_{2}^{+}$с электронами по уровням конфигурации $2 p^{5} 3 d$ атома неона в послесвечении диэлектрического барьерного разряда низкого давления. Впервые измерения проводились в условиях предельно низких (менее 1 Torr) давлений газа, исключающих влияние неупругих атом-атомных столкновений на населенности возбужденных уровней. Результаты работы показывают необходимость анализа каскадных $4 p \rightarrow 3 d$ переходов, неопределенность вклада которых в заселение $3 d$-уровней не позволяет отождествить полученные данные с парциальными коэффициентами диссоциативной рекомбинации, характеризующими распределение потока рекомбинации по выходным каналам процесса. Сопоставление потоков фотонов на переходах с уровней $4 p_{5}$ и $3 d_{11}$, связанных инфракрасным переходом, позволяет предположить, что механизм диссоциативной рекомбинации $\mathrm{Ne}_{2}^{+}$таков, что его выходные каналы не содержат уровней конфигурации $2 p^{5} 3 d$.

\section{Конфликт интересов}

Автор заявляет, что у него нет конфликта интересов.

\section{Список литературы}

[1] Bates D.R., Massey H.S.W. // Proc. Roy. Soc. (London). 1947. V. A192. P. 1.

[2] Mihajlov A.A., Ignjatović L.M., Dimitrijević M.S., Djurić Z. // Astrophys. J. Suppl. S. 2003. V. 147. N 2. P. 369. doi $10.1086 / 375621$

[3] Friedl R., Rauner D., Heiler A., Fantz U. // Plasma Sources Sci. Technol. 2020. V. 29. N 1. P. 015014. https://doi.org/10.1088/1361-6595/ab5ae5

[4] Ivanov V.A., Skoblo Yu.E. // Opt. Spectrosc. 2019. V. 127. N 5. P. 820. doi 10.1134/S0030400X19110110

[5] Lebedev V.S., Kislov K.S., Narits A.A. // Plasma Sources Sci. Technol. 2020. V. 29. N 2. P. 025002.

https://doi.org/10.1088/1361-6595/ab652f
[6] Bates D.R. // J. Phys. B: At. Mol. Phys. 1979. V. 12. N 1. P. L35. https://doi.org/10.1088/0022-3700/12/1/008.

[7] Frommhold L., Biondi M.A., Meir F.J. // Phys. Rev. 1968. V. 165. N 1. P. 44.

[8] Sauter G.F., Gerber R.A., Oskam H.J. // Physica. 1966. V. 32. P. 1921.

[9] Veatch G.E., Oskam H.J. // Phys. Rev. V. A2. N 4. P. 1422.

[10] Connor T.R., Biondi M.A. // Phys. Rev. 1965. V. 140. N 3A. P. 778.

[11] Steenhuijsen L.W.G., Van Schaik N., Van de Nieuwenhuyzen L.C.A.M., Verspaget F.H.P. // J. Phys. Colloq. 1979. V. 40. P. C7.

[12] Malinovský L., Lukač P., Hong J. // Czech. J. Phys. 1986. V. 36. P. 1035.

[13] Malinovský L., Trnovec J., Hong C.J., Tálsky A. // Czech. J. Phys. 1990. V. 40. P. 191.

[14] Гордеев С.В., Иванов В.А., Скобло. Ю.Э. // Опт. и спектр. 2019. T. 127. № 3. C. 247.

doi 10.21883/OS.2019.09.48190.106-19; Gordeev S.V., Ivanov V.A., Skoblo Yu.E. // Opt. Spectrosc. 2019. V. 127. N 3. P. 418.

[15] Mulliken R.S. // Phys. Rev. 1964. V. 136 N 4A. P. 962.

[16] Bates D.R. // Adv. At. Mol. Phys. Eds. 1979. V. 15. P. 235.

[17] Gurevich A.V., Pitaevskii L.P. // Sov. Phys. JETP. 1964. V. 19. N 4. P. 870 .

[18] Dahler J.S., Franklin J.L., Munson M.S.B., Field F.H. // J. Chem. Phys. 1962. V. 36. N 12. P. 3332. https://doi.org/10.1063/1.1732466

[19] Иванов В.А. // Опт. и спектр. 1991. V. 70. № 5. Р. 67.

[20] Ivanov V.A. // Plasma Sources Sci. Technol. 2020. V. 29. N 4. P. 045022. https://doi.org/10.1088/1361-6595/ab7f4c

[21] Holstein T. // Phys. Rev. 1947. V. 72. N 12. P. 1212.

[22] Holstein T. // Phys. Rev. 1953. V. 83. N 6. P. 1159.

[23] Frommhold L., Biondi M.A. // Phys. Rev. 1969. V. 185. N 1. P. 244.

[24] Lilly R.A. // J. Opt. Soc. Am. 1976. V. 66. N 3. P. 245.

[25] Emmons D., Weeks D.E., Eshel B., Perram G.P. // J. Appl. Phys. 2018. V. 123. P. 043304. https://doi.org/10.1063/1.5009337

[26] Ngassam V., Orel A.E. // Phys. Rev. A. 2006. V. 73. N 3. P. 032720. doi 10.1103/PhysRevA.73.032720

[27] Royal J., Orel A.E. // Phys. Rev. A. 2006. V. 73. N 4. P. 042706. doi 10.1103/PhysRevA.73.042706 